\title{
Relation among body mass index, waist-hip ratio, and pulmonary functional residual capacity in normal weight versus obese Chilean children: A cross-sectional study
}

\author{
Rodrigo Muñoz Cofré, MSc. ${ }^{a, b}$, Mariano del Sol, M.D. ${ }^{a}$, Paul Medina González, MSc. ${ }^{c}$, \\ Javiera Escobar Inostroza, B.S. ${ }^{d}$, Pablo A. Lizana, M.D. ${ }^{e}$, Daniel Conei, B.S. ${ }^{a, f}$ and \\ Máximo Escobar Cabello, MSc. ${ }^{b, c}$
}

\begin{abstract}
Introduction. Obesity is associated with a rapid decrease in ventilatory function. The most common way of assessing nutritional status and measuring abdominal fat and hips are the body mass index (BMI) and the waist-hip ratio (WHR). There is scarce evidence suggesting their relation to functional residual capacity (FRC). Our objective was to determine the relation among BMI, WHR, and FRC in obese children in the city of Talca, Chile.
\end{abstract}

a. Doctoral Program in Morphological Sciences, Universidad de la Frontera, Temuco, Chile.

b. Laboratory of Ventilatory FunctionDysfunction.

c. Department of Kinesiology.

d. Master's Program in Kinesiology, Universidad Católica del Maule, Talca, Chile.

e. Laboratory of Morphological Sciences, Institute of Biology, Pontificia Universidad Católica de Valparaíso, Valparaíso, Chile.

f. Department of Morphological Sciences, School of Science, Universidad San Sebastián, Puerto Montt, Chile.

E-mail address:

Rodrigo Muñoz Cofré, MSc.: rodrigomunozcofre@ gmail.com

Funding:

None.

Conflict of interest:

None.

Received: 6-22-2018

Accepted: 12-12-2018

\section{INTRODUCTION}

The World Health Organization (WHO) has defined obesity as "an abnormal or excessive fat accumulation that presents a risk to health." Obesity has increased alarmingly in developing countries. ${ }^{1}$ In Chile, childhood obesity has continued increasing, especially since 2009, after the economic crisis. ${ }^{2,3}$ The latest data by the National Ministry of Health of Chile $(\mathrm{MoH})$ show that, in children younger than 6 years, obesity accounted for $9.6 \%$ in 2007 and reached $9.9 \%$ in $2010 .{ }^{4}$

Fat mass is the most variable component of body composition and there are risks associated with excess fat body mass and its location. ${ }^{5}$ This way, at present, it has been confirmed that the metabolic and mechanical consequences of regional fat distribution are particularly relevant for clinical examination and for actions to be taken in this regard. ${ }^{6}$

One of the many problems associated with childhood and adolescent obesity is the rapid decrease in ventilatory function, and the mechanical and inflammatory processes of this condition lead to a reduced chest and lung performance ${ }^{7}$ and to changes in respiratory physiology, the mechanics of ventilation, and respiratory muscles, ${ }^{8}$ thus reducing chest expansion and airway caliber. ${ }^{9}$ Hence, a reduced functional residual capacity (FRC), which is the volume of air present in the lungs at the end of passive expiration, is a typical characteristic of obesity hypoventilation syndrome. ${ }^{10}$ 
This results from a change in inspiratory and expiratory pressures due to the increase in fat deposit in the chest and abdomen. In addition, the increase in intra-abdominal pressure is transferred to the chest, resulting in changes in the pressure-volume curve, with the consequent decrease in static lung volumes. ${ }^{6,9}$ However, there are few precedents on the consequences of fat tissue location and its relation to FRC, considering that this concept has historically been related to heart problems. ${ }^{11}$

The existence of a reverse relation between FRC and body mass index (BMI) has been documented; ${ }^{6}$ the latter is an overall measure of body mass that includes both fat and lean body mass, without establishing the differences in fat tissue distribution in the chest and abdomen. ${ }^{2}$ This is relevant due to the fact that the reduced lung volumes in obesity are the result of the mechanical -direct or indirect- effect of fat on the rib cage. ${ }^{6,9}$ In this context, one of the most common manners to measure abdominal and hip fat is the waist-hip ratio (WHR). This is an outcome measure of fat tissue distribution and the most commonly used measure in public health settings. However, it has been mainly studied in association with risk factors and cardiovascular disease. ${ }^{11}$ It has been observed that there is a direct relation between extra- and intraabdominal fat, ${ }^{8}$ which would cause respiratory imbalance and alter diaphragm effectiveness and, as a consequence, lung volumes. ${ }^{6,9}$ However, WHR has not been considered an indicator of FRC problems in obese individuals. In this manner, this study would contribute to demonstrate that this index may predict respiratory alterations associated with obesity.

Based on the information presented here, we may hypothesize that an increase in BMI and WHR is related to a decreased FRC in obese children. The objective of this study was to determine the relation among BMI, WHR, and FRC in obese children aged 6-12 years in the city of Talca, Chile.

\section{POPULATION AND METHODS}

This was a cross-sectional study carried out between March and May 2017 in the city of Talca. The sample size was estimated using the Ene 3.0 ${ }^{\circledR}$ software for sample size estimation (Barcelona, Spain).

Based on a pilot study done in 10 normal weight and 10 obese children, and considering a 0.95 significance level, an $80 \%$ statistical power,
2 groups, a $10 \%$ dropout, and a 2.19 mean and a 0.55 liter standard deviation in FRC, the sample was determined at a total of 36 children aged 6-12 years in the city of Talca. ${ }^{12}$ The Talca population was 220000 inhabitants; of these, 30028 were $5-14$ years old; in turn, $11.79 \%$ of them were obese (http: / / www.deis.cl /).

Using a non-probability sampling, children were selected from schools. In a meeting with children's guardians, the project was presented and the names of parents who agreed to participate were recorded. Subsequently, an appointment for assessment was made on the telephone. Based on BMI, children were divided into 2 groups: normal weight, those who had a BMI-for-age within the standard deviation established by the median value according to the WHO Child Growth Standards $(\mathrm{n}=18)$, and obese, those with a BMI-for-age with more than 1 standard deviation above the median value established as per the WHO Child Growth Standards ${ }^{1}(\mathrm{n}=18)$. Pulmonary function tests were done at the Laboratory of Ventilatory Function-Dysfunction of Universidad Católica del Maule by the kinesiologist in charge (certified by the MoH). Children had to attend the test with their guardians. Inclusion criteria were age in the age range and being normal weight or obese, whereas exclusion criteria were incompatible physical and / or cognitive status, acute or chronic respiratory disease, girls who had experienced the menarche or had nausea and vomiting. This study was approved by the Scientific Ethics Committee of Universidad Católica del Maule (Resolution 23/2016). Parents or legal guardians were asked to sign the informed consent and, before starting the assessment, children were asked for their assent. Results were delivered to parents and they were asked to take them to their children's next health checkup. Lastly, data were maintained strictly confidential and were coded to safeguard participants' identity.

\section{Anthropometric measures and indices}

Height: The distance from the floor to the vertex was measured using a SECA ${ }^{\circledR}$ anthropometer (model 220, Hamburg, Germany). The subject was standing and barefoot, with the heels together and the toes pointing out to a 45-degree angle. The heels, the buttocks, the back, and the occipital region had to be in contact with the surface of the anthropometer. The measurement was done during a maximum inspiration and keeping the head adjusted to 
the Frankfurt plane. ${ }^{13}$ The value was recorded in meters.

Body weight: Weight was measured in kilograms using a SECA ${ }^{\circledR}$ scale (model 840, Hamburg, Germany). ${ }^{13}$

BMI: This is an indicator of the weight and height relation; it was used to identify overweight and obesity. It was obtained by dividing the weight in kilograms by the square of the height in meters $\left(\mathrm{kg} / \mathrm{m}^{2}\right){ }^{1}$

Waist circumference: This was measured with the subject relaxed, standing, and with the arms on the chest. It was taken at the end of a normal exhalation at the narrowest point between the last rib and the iliac crest or, failing this, at the midline if the narrowest point could not be established. ${ }^{13}$ The value was recorded in centimeters.

Hip circumference: This was measured with the subject relaxed, standing, and with the arms crossed on the chest. It was taken at the end of a normal exhalation at the maximum circumference at the level of the buttocks, corresponding to the symphysis pubis. ${ }^{13}$

WHR: This is an anthropometric measure used to determine the level of intra-abdominal fat. It was estimated as the ratio between waist circumference and hip circumference. ${ }^{1}$

Lung volumes: Tests were done using a Medgraphics body plethysmograph (Platinum Elite DL®, St. Paul, Minnesota, USA). It was measured as per the American Thorax Society (ATS) standards. The subject was sitting down, with the mouthpiece at the level of the oral cavity, the lips tightly closed around it during the test to prevent air leak, wearing a nasal clip and with the hands on the cheeks to support the facial muscles. Then, the booth was closed and the subject was asked to breath 4 times at tidal volume. The subject was asked to "pant softly" trying to move volumes between 50 and $60 \mathrm{~mL}$ while supporting the cheeks with the tip of the fingers to prevent mouth pressure fluctuations. The frequency of panting had to be close to 60 per minute $(1 \mathrm{~Hz})$. The provider in charge activated the shutter for 2-3 seconds; then he/she asked the subject to inhale to the maximum level and then exhale until reaching residual volume (RV). ${ }^{14}$ The best value out of three measurements was selected, with a $5 \%$ variability in total lung capacity (TLC). FRC was recorded in liters.

Statistical analysis: The GraphPad Prism statistical software was used (version $5.0^{\circledR}$, San Diego, USA). Outcome measures were described as average \pm standard deviation.
To establish the differences in the behavior of expiratory reserve volume (ERV), RV, and FRC as per the nutritional status of normal weight versus obese children, Student's t test or the MannWhitney U test were used, according to outcome measure distribution. To establish a correlation among BMI, WHR, and FRC, Pearson's or Spearman's $r$ test was done, depending on data distribution. In this regard, charts were developed as per nutritional status. The statistical significance level was established at $p<0.05$.

\section{RESULTS}

Out of 240 guardians who were invited to participate from 6 courses, the first 18 normal weight children and the first 18 obese children were selected; they corresponded to 15 girls and 21 boys aged 6-12 years. The characteristics of the sample are shown in Table 1. The comparison between groups showed a significant difference in the WHR, ERV, and FRC outcome measures between obese and normal weight children (Table 1). A significant difference was observed in weight, BMI $(p=0.0001)$ and WHR $(p=0.022)$ in obese children compared to normal weight ones; on the contrary, there was a significant difference between ERV $(p=0.006)$ and FRC $(p=0.025)$ of obese children compared to normal weight ones (Figure 1). In addition, a direct (moderate and significant) relation was found between BMI and FRC in normal weight children $(\mathrm{r}=0.681 ; p=0.03)$ (Figure 2). Lastly, there was a reverse relation between WHR and FRC, which was moderate in normal weight children $(\mathrm{s}=-0.489 ; p=0.03)$ and high in obese children $(\mathrm{r}=-0.681 ; p=0.001)$ (Figure 3).

\section{DISCUSSION}

The objective of this study was to determine the relation among BMI, WHR, and FRC in obese children aged 6-12 years in the city of Talca, Chile. Results showed that there was a significant reduction in the FRC of obese children, and also a reverse and high relation between WHR and FRC. The relevance of this lies in detecting the systemic effects caused by obesity and central fat distribution at an early age. This reinforces the usefulness of BMI and introduces WHR as an indicator of both cardiovascular disease $\mathrm{e}^{2,3}$ and ventilatory function in children. ${ }^{11}$

Specifically, central fat tissue accumulation would reduce chest wall elastance, which would, in turn, decrease FRC and ERV and generate an early reduction in expiratory flow during 
TABLE 1. Anthropometric characteristics of normal weight and obese children

\begin{tabular}{lccc}
\hline Outcome measures & Normal weight & Obese & p value \\
\hline Total sample & 18 & 18 & - \\
Sex (M-F) & $14 / 4$ & $7 / 11$ & - \\
Age (years old) & $10 \pm 2$ & $9 \pm 2$ & $0.257^{\mathrm{a}}$ \\
Weight (kg) & $34.36 \pm 6.39$ & $49.21 \pm 12.61$ & $0.0002^{\mathrm{b}}$ \\
Height (m) & $1.41 \pm 0.11$ & $1.38 \pm 0.11$ & $0.759^{\mathrm{a}}$ \\
BMI (kg/m $\left./ \mathrm{m}^{2}\right)$ & $17.12 \pm 1.17$ & $25.50 \pm 3.26$ & $0.0001^{\mathrm{b}}$ \\
WHR & $0.86 \pm 0.08$ & $0.91 \pm 0.04$ & $0.022^{\mathrm{b}}$ \\
ERV (L) & $0.91 \pm 0.44$ & $0.60 \pm 0.30$ & $0.006^{\mathrm{b}}$ \\
RV (L) & $1.08 \pm 0.50$ & $0.81 \pm 0.47$ & $0.107^{\mathrm{b}}$ \\
FRC (L) & $1.99 \pm 0.67$ & $1.56 \pm 0.41$ & $0.025^{\mathrm{a}}$ \\
\hline
\end{tabular}

WHR: waist-hip ratio; ERV: expiratory reserve volume; RV: residual volume; FRC: functional residual capacity; a: Mann-Whitney; b: Student's $t$ test.
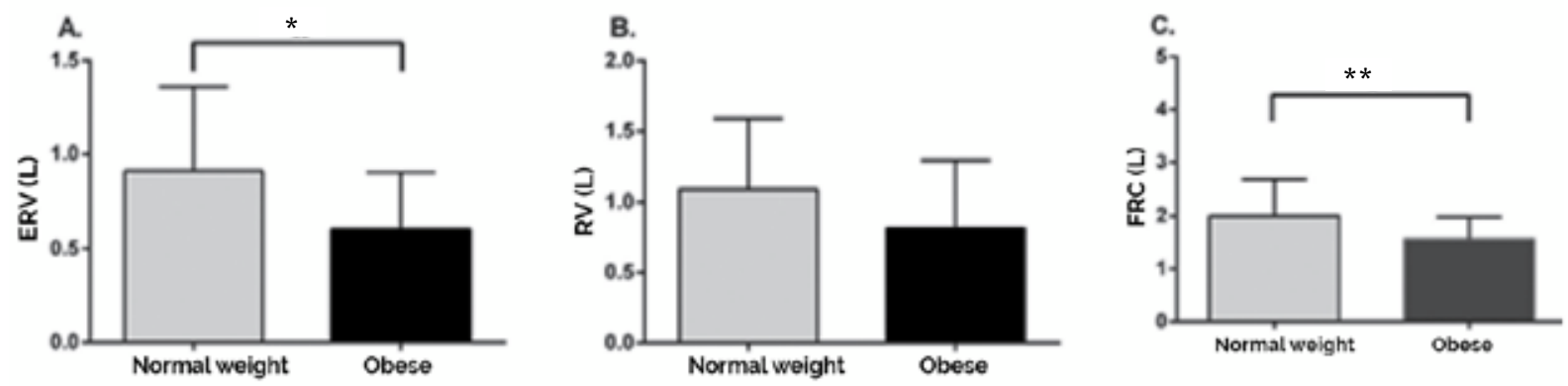

ERV: expiratory reserve volume; RV: residual volume; FRC: functional residual capacity.

A: Comparison of ERV between normal weight and obese children. Statistical test, Mann-Whitney;

B: Comparison of RV between normal weight and obese children. Statistical test, Student's $t$ test;

C: Comparison of FRC between normal weight and obese children.

Statistical test, Student's $t$ test. ${ }^{*}=0.004{ }^{* *}=0.03$.
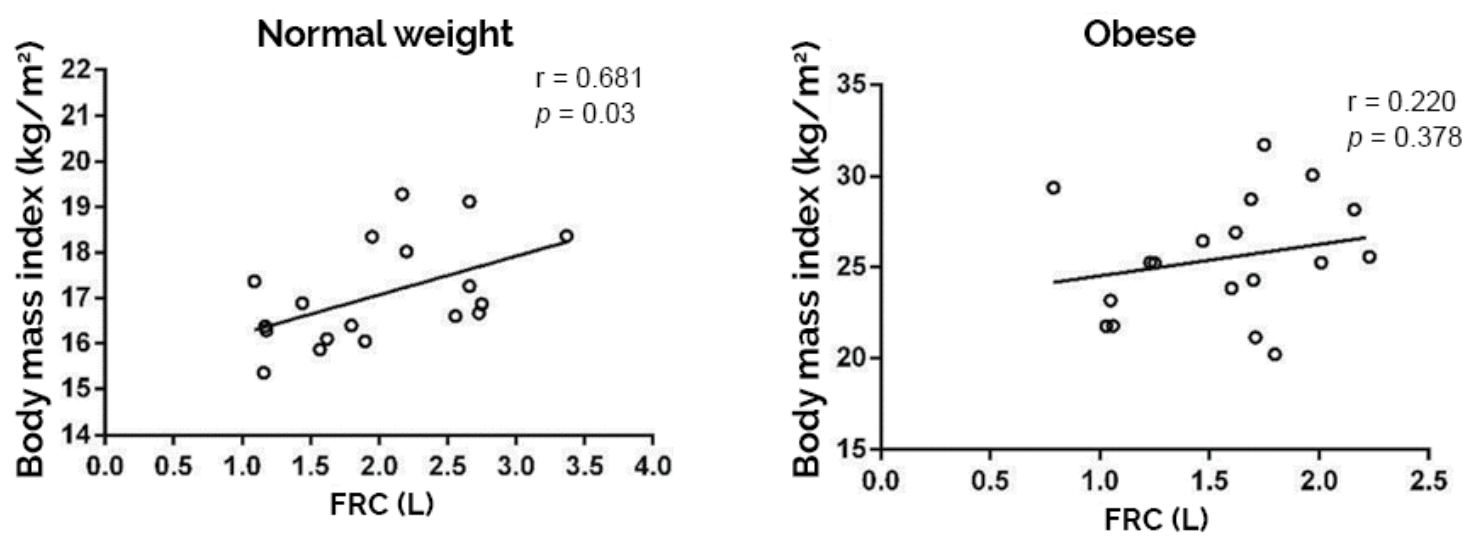

FRC: functional residual capacity; r: Pearson. 
breathing at tidal volume, especially at the lung bases. This has been reinforced by studies that evidenced a reverse relation between FRC and airway resistance in obese subjects. ${ }^{6,9}$ This is supported by Wilson's three-compartmental model, ${ }^{15}$ where the abdomen is considered to be associated with the lower chest and, therefore, may have a positive or negative effect on the diaphragmatic activity through the abdominal wall. ${ }^{16,17}$ The role of the latter on the ventilatory pump has been described in physical models as a passive agent that would impact on the concentric contraction of the diaphragm. However, when such compartment is not normal, as in the case of obesity, for example, ventilation may be affected, thus reducing FRC, among other variables.

The results of this study reassert prior findings in the adult and elderly population, which showed an association between pulmonary function and anthropometric outcome measures, ${ }^{15-18}$ specifically, a reduced pulmonary function with an increase in waist circumference ${ }^{17}$ and WHR, ${ }^{18-21}$ considered better predictors than BMI. ${ }^{18,21}$ Ceylan et al. studied the prevalent abnormality of pulmonary function in overweight and obese subjects, and the correlation between a worsened pulmonary function and the degree of obesity. To this end, 31 women and 22 men with a mean age of 40 years were studied. A reduction in FRC and ERV was observed in overweight and obese subjects. In addition, WHR and BMI were better predictors of ERV in women, whereas only WHR did in men. Based on this, it was concluded that lung volumes were affected by the distribution of body fat..$^{22}$ This was consistent with our results, which showed a significant reduction in FRC and ERV in obese children, compared to normal weight ones. It is worth noting that our sample was made up of pediatric subjects and no differences between sexes were analyzed.

BMI use for the characterization of pulmonary function has been controversial because it considers both lean and fat body mass, which hinders the determination of an abdominal effect on an effective ventilatory mechanics. ${ }^{21,23}$ For example, the assessment of pulmonary function with a spirometry in the pediatric obese population based on BMI has demonstrated dissimilar results, and there is evidence that a higher BMI reduces forced vital capacity and forced expiratory volume in 1 second. ${ }^{24}$ Besides, no significant differences were observed in the same outcome measures when comparing obese and normal weight children. ${ }^{23,25}$

The results of ERV and FRC in obese children demonstrated that BMI was sensitive to changes in pulmonary function in the studied sample. In pediatrics, this may be because this index has a positive correlation with fat tissue and may also be associated with risk factors for noncommunicable chronic diseases. ${ }^{26}$ In this setting, the combined analysis of BMI and WHR would be the most advisable option to determine potential respiratory system disorders. ${ }^{27}$ It is worth noting that no differences were observed in RV; this was consistent with what has been reported in the bibliography, which indicated that this outcome measure was maintained. ${ }^{24}$

Besides, our sample was made up of pediatric subjects without history of respiratory

FIGURE 3. Relation between waist-hip ratio and functional residual capacity in normal weight and obese children
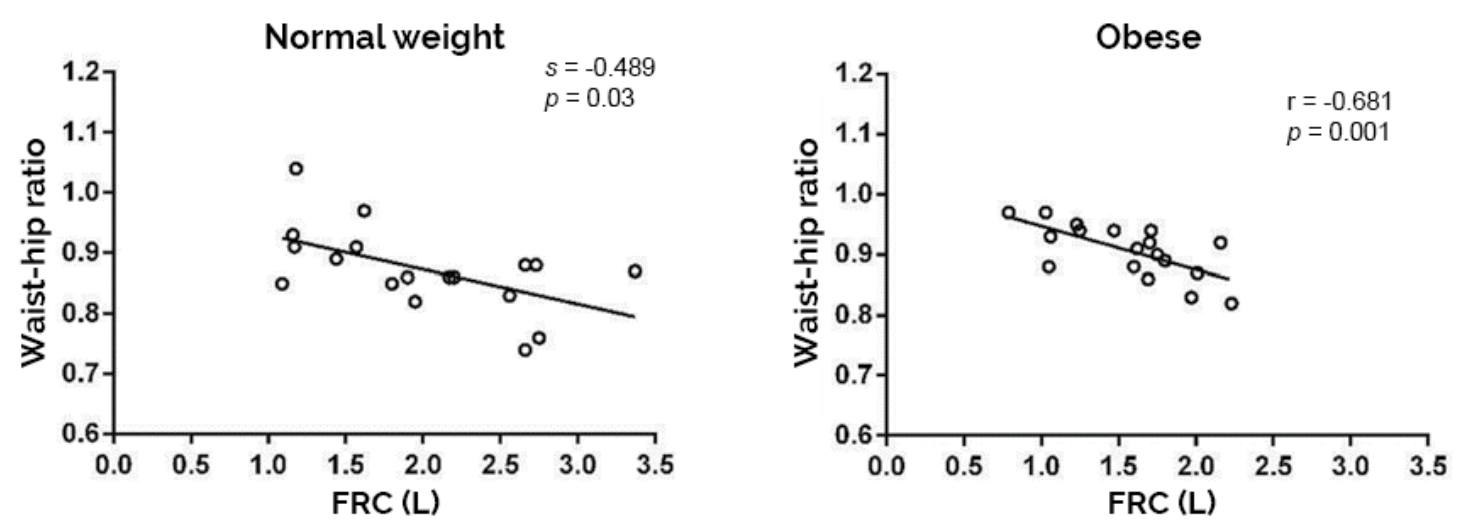

CRF: capacidad residual funcional; s: Spearman; r: Pearson. 
disorders; therefore, there is no lung parenchyma compromise, as observed in adult patients with chronic obstructive disease. In the latter case, the early closure of small-caliber airways results in an increased RV and a limited expiratory flow, which, as a whole, causes an increase in FRC. ${ }^{12,28}$

Lastly, it is worth pointing out that our subjects were children who had not reached physical and respiratory maturity. In addition, the understanding of the test varied among children depending on their maturity stage, which differentiated them from adults, and this had an effect on the performance of pulmonary function tests. ${ }^{29}$

\section{Limitations}

This study had a series of limitations. As mentioned above, the sample was not analyzed in terms of sex, so different correlations could arise, but this was not part of our initial objective. Future studies should include this outcome measure in larger samples. Due to aspects related to methodology and sample size, the results reported here were only applied to the assessed group and cannot be generalized. Notwithstanding this, the results were consistent with those of other authors, making the data found here in relation to obese children and their FRC relevant.

\section{CONCLUSIONS}

Our results indicated that FRC decreased significantly in obese children, compared to normal weight ones. In addition, a higher WHR was reported to reduce FRC, which turned WHR into an important tool for physical education teachers, kinesiologists, and health care providers as an indicator of potential ventilatory dysfunction in childhood.

\section{REFERENCES}

1. Organización Mundial de la Salud. Sobrepeso y obesidad infantiles. [Accessed on: February $23^{\text {rd }}, 2018$ ]. Available at: http: / / www.who.int/dietphysicalactivity/childhood/ es/.

2. Lizana PA, Paula CV, Araya L, Aguilera F, et al. Obesity, Body Fat Distribution, and Physical Activity in School-age Children: an Urban and Rural Comparison in Valparaíso, Chile. Biomed Environ Sci. 2016; 29(11):834-39.

3. Lizana PA, González S, Lera L, Leyton B. Association between body composition, somatotype and socioeconomic status in Chilean children and adolescents at different school levels. J Biosoc Sci. 2018; 50(1):53-69.

4. Mönckeberg F, Muzzo S. La desconcertante epidemia de obesidad. Rev ChilNutr. 2015; 42(1):96-102.

5. Xanthopoulos M, Tapia IE. Obesity and common respiratory diseases in children. Paediatr Respir Rev. 2017; 23:68-71.

6. Peters U, Suratt BT, Bates JHT, Dixon AE. Beyond BMI:
Obesity and Lung Disease. Chest. 2018; 153(3):702-9.

7. Ogilvie RP, PatelSR. The epidemiology of sleep and obesity. Sleep Health. 2017; 3(5):383-8.

8. Feng K, Chen L, Han S, Zhu GJ. Ratio of waist circumference to chest circumference is inversely associated with lung function in Chinese children and adolescents. Respirology. 2012; 17(7):1114-8.

9. Mafort TT, Rufino R, Costa CH, Lopes AJ.Obesity: systemic and pulmonary complications, biochemical abnormalities, and impairment of lung function. Multidiscip Respir Med. 2016; 11:28.

10. Lin CK, Lin CC. Work of breathing and respiratory drive in obesity. Respirology. 2012; 17(3):402-11.

11. Rosales R. Antropometría en el diagnóstico de pacientes obesos; una revisión. Nutr Hosp. 2012; 27(6):1803-9.

12. Parraguez Arévalo A, Rojas Navarro F, Ruz Céspedes M, Medina González P, et al. Influencia de la obesidad sobre la resistencia y conductancia específica de la vía aérea en niños escolares. Arch Argent Pediatr. 2018; 116(2):e227-33.

13. International Society for the Advancement of Kinanthropometry. International Standards for Anthropometric Assessment. Underdale: ISAK; 2001.

14. Wanger J, Clausen JL, Coates A, Pedersen OF, et al. Standardisation of the measurement of lung volumes. Eur Respir J. 2005; 26(3):511-22.

15. Wilson TA. Compartmental models of the chest wall and the origin of Hoover's sign. Respir Physiol Neurobiol. 2015; 210:23-9.

16. De Troyer A, Boriek AM. Mechanics of the Respiratory Muscles. Compr Physiol. 2011; 1(3):1273-300.

17. Talasz H, Kremser C, Kofler M, Kalchschmid E, et al. Phase-locked parallel movement of diaphragm and pelvic floor during breathing and coughing-a dynamic MRI investigation in healthy females. Int Urogynecol J. 2011; 22(1):61-8.

18. Santana H, Zoico E, Turcato E, Tosoni P, et al. Relation between body composition, fat distribution, and lung function in elderly men. Am J Clin Nutr. 2001; 73(4):827-31.

19. Canoy D, Luben R, Welch A, Bingham S, et al. Abdominal Obesity and Respiratory Function in Men and Women in the EPIC-Norfolk Study, United Kingdom. Am J Epidemiol. 2004; 159(12):1140-9.

20. Chen Y, Rennie D, Cormier YF, Dosman J. Waist circumference is associated with pulmonary function in normal-weight, overweight, and obese subjects. Am J Clin Nutr. 2007; 85(1):35-9.

21. Sable M, Kowale AN, Aundhkar NV, Sable S. The Effect of Gender On The Relationship Between Body Fat Distribution \& Lung Functions In Adult Indian Population. IOSR-JDMS. 2016; 15(3):73-7.

22. Ceylan E, Cömlekçi A, Akkoçlu A, Ceylan C, et al. Theeffects of body fat distribution on pulmonary function tests in the overweight and obese. South Med J. 2009; 102(1):30-5.

23. LiyanageG, JayamanneBD, Aaqiff M, Sriwardhana D. Effect of body mass index on pulmonary function in children. Ceylon Med J. 2016; 61(4):163-6.

24. Fretzayas A, Moustaki M, Loukou I, Douros K. Is obesity related to the lung function of non-asthmatic children? World J Clin Pediatr. 2018; 7(2):67-74.

25. Tenório LH, Santos AC, De Oliveira AS, De Lima AM, et al. Obesity and pulmonary function tests in children and adolescents: a systematic review. Rev Paul Pediatr. 2012; 30(3):423-30.

26. Boran P, Tokuc G, Pisgin B, Oktem S, et al. Impact of obesity on ventilatory function. J Pediatr (Rio J). 2007; 83(2):171-6.

27. Kaufer- Horwitz M, Toussaint G. Indicadores antropométricos para evaluar sobrepeso y obesidad en pediatría. Bol Med Hosp Infant Mex. 2008; 65(6):502-18. 
236 / Arch Argent Pediatr 2019;117(4):230-236 / Original article

28. Lufti MF. The physiological basis and clinical significance of lung volume measurements. Multidiscip Respir Med. 2017; 12:3.
29. Orliaguet G, Riou B, Leguen M. Maturation postnatale du diaphragme: des modifications ultrastructurales aux modifications fonctionelles. Ann Fr Anesth Reanim. 2004; 23(5):482-94. 